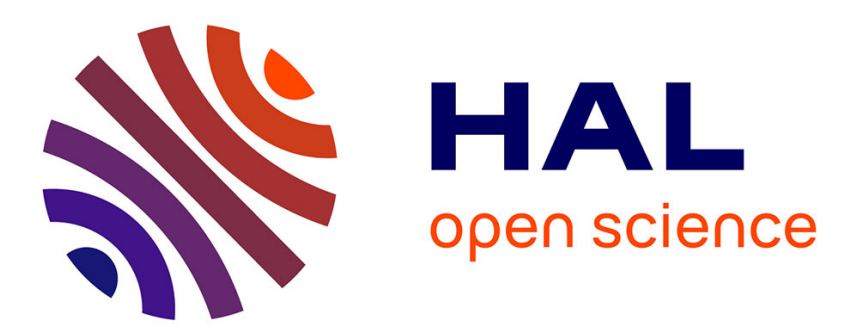

\title{
Active optical-based detuning circuit for receiver endoluminal coil
}

I Saniour, R. Aydé, A-L Perrier, G Gaborit, L Duvillaret, R Sablong, O Beuf

\section{To cite this version:}

I Saniour, R. Aydé, A-L Perrier, G Gaborit, L Duvillaret, et al.. Active optical-based detuning circuit for receiver endoluminal coil. Biomedical Physics \& Engineering Express, 2017, 3 (2), 10.1088/20571976/aa5db0 . hal-01492605

\section{HAL Id: hal-01492605 https://hal.science/hal-01492605}

Submitted on 19 Jun 2018

HAL is a multi-disciplinary open access archive for the deposit and dissemination of scientific research documents, whether they are published or not. The documents may come from teaching and research institutions in France or abroad, or from public or private research centers.
L'archive ouverte pluridisciplinaire HAL, est destinée au dépôt et à la diffusion de documents scientifiques de niveau recherche, publiés ou non, émanant des établissements d'enseignement et de recherche français ou étrangers, des laboratoires publics ou privés. 


\title{
Active Optical-Based Detuning Circuit for Receiver Endoluminal Coil
}

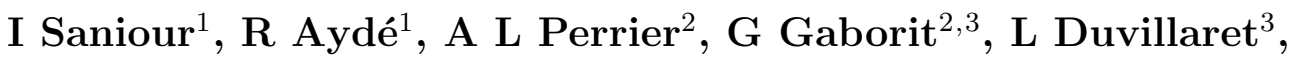 \\ R Sablong ${ }^{1}$ and O Beuf ${ }^{1}$ \\ ${ }^{1}$ Centre de Recherche en Acquisition et Traitement de l'Image pour la Santé \\ (CREATIS), Université de Lyon - CNRS UMR 5220 - INSERM U1206 - Université \\ Lyon 1 - INSA Lyon - Université Jean Monnet Saint-Etienne, 69616 Villeurbanne, \\ France \\ 2 Institut de Microélectronique, Electromagnétisme et Photonique et le Laboratoire \\ d'Hyperfréquence et de Caractérisation (IMEP-LAHC), Université de Savoie, 73376 \\ Le Bourget-du-Lac, France \\ 3 Kapteos, 73800 Sainte-Hélène-du-Lac, France \\ E-mail: olivier.beuf@creatis.insa-lyon.fr
}

\begin{abstract}
In this paper we demonstrate the effectiveness of an active optical detuning circuit for magnetic resonance imaging (MRI) endoluminal receiver coil. Three endoluminal coils prototypes were built: a coil without any detuning circuit, a coil with a galvanic (classic) detuning circuit using a PIN diode, and a coil with an optical detuning circuit using two photodiodes in parallel with a PIN diode. These coils were built and characterized on a laboratory experimental bench. Then, an in vitro experiment was performed with a $3.0 \mathrm{~T}$ MR system to evaluate the impact of the endoluminal receiver coils in detuned phase on the image uniformity distribution measured using the body coil. Next, the endoluminal coil was used as a receiver coil to compare the signal-to-noise ratio (SNR) distribution based on iso-contour maps. On experimental bench, the results show an increase delay of the switching times (tuned-detuned or detuned-tuned) for optical-detuned coils of about $10 \mu \mathrm{s}$ due to the electro-optical circuits, delay still compatible with requirements. When the body coil is used as a transceiver, the SNR uniformity is similar whether the galvanic or the optical detuning circuit is used. Finally, the SNR iso-contours of the different endoluminal coils prototypes are comparable.
\end{abstract}

Keywords: magnetic resonance imaging, optical detuning, endoluminal coil, fibered sensor, galvanic link, photodiodes.

\section{Introduction}

Magnetic resonance imaging (MRI) is widely used to diagnose and analyze inflammatory bowel diseases (Geenen et al 2007) and to determine rectal cancer stages (Beets et al 2013, Dewhurst et al 2013, Torkzad et al 2010). However, accurate exploration of bowel diseases and detailed information about the colon wall layers, mandatory for proper 
management and effective treatment, are still not available. Indeed, despite the huge progresses in radiofrequency $(\mathrm{RF})$ coils, based on the phased array technology with increase of channels (Giusti et al 2012), the external MRI coils have remained unable to provide adequate SNR to reach required high spatial resolution images. By comparison several authors have demonstrated that internal coils significantly increase the local SNR and makes possible either to perform fast in vivo MR spectroscopy of heart tissues or to obtain high spatial resolution MR images of colon walls in preclinical context (Beuf et al 2004, Dorez et al 2016, Kantor et al 1984, Pilleul et al 2005, Ramgolam et al 2011).

However, for clinical use, conducting coaxial cables linking the coil (deeply introduced into the patient) to the connecting plug of the MR system may induce safety issues. In fact, the electrical field $\vec{E}$, accompanying the RF magnetic field $\overrightarrow{B_{1}}$, induces currents in the conducting cables at the same frequency that can lead to a strong increase of the local specific absorption rate (SAR) by the tissues (Detti et al 2011, Yeung et al 2002). Different solutions were proposed to diminish the SAR issue, i.e., coaxial chokes (Ladd et al 2000), transformer coupled transmission lines (Weiss et al 2005), baluns matching circuits, and traps (Peterson et al 2003). These methods do not ensure a total safety for the patient. The use of optical fibers instead of coaxial cables appears as an attractive alternative to solve definitely safety issues. Different solutions have already been explored to ensure an optical connection between MRI and some coils (Fandrey et al 2012, Koste et al 2005, Memis et al 2008, Possanzini et al 2011, Yuan et al 2007). For example in reference (Possanzini et al 2011), the dStream ${ }^{\mathrm{TM}}$ architecture of Philips has introduced a hybrid technique to amplify, filter, and sample the detected nuclear magnetic resonance (NMR) signal to a numerical one to be then converted into an optical signal and transmitted using an optical fiber. In this latter case, the active detuning is classically performed using coaxial cables. Regarding endoluminal coils, whereas the major issue is the optical conversion and transmission of the NMR signal, the optical tuning/detuning is also an important issue to have a fully optical receiver coil. The receiver coil has to be detuned during RF transmit phase to avoid the strong coupling between coils and $\vec{B}_{1}$ concentrations close to the receiver coil, which affects the image uniformity and involve again the SAR issues due to E-field concentrations. Thus in the RF-transmission phase, the endoluminal coil should be detuned from the resonance frequency to ensure isolation between transmit and receive coils. PIN diodes are usually used for passive or/and active detuning of the receiver coils. In passive detuning, a crossed pair of fast switching PIN diodes is added to the resonant circuit. When the RF field is transmitted, the induced current alternatively switches one of the diodes within the circuit. Although it is a wireless method, passive detuning is not sufficiently effective because, depending on RF pulses used, the induced current is not always sufficient to activate the PIN diode which can degrade again image uniformity. Moreover, the high conduction losses in fast switching diodes may induce for endoluminal coil considerable heat inside patient's body (Burl et al 2005). On the other hand, active detuning can be done by using a PIN diode added to the resonant circuit. The MR system transmits a direct current (DC) to activate the PIN diode 
when the $\overrightarrow{B_{1}}$ field is emitted. Hence the low impedance of the PIN diode changes the self-resonant frequency of the coil. This method is faster, more reliable and safer than the passive detuning methods. Conventionally, the DC bias current is provided by the MR system and transmitted using a coaxial cable. For some specific applications, optical active detuning was already carried out. For instance, a safe, reliable and precise catheter tracking was demonstrated for intervention MRI procedures (Weiss et al 2004, Wong et al 2000). But those studies were not used for imaging and did not require an optical detuning system with high switching performance. Korn et al have used optical detuning for coils dedicated to compensate the physiological motion (Korn et al 2011). The switching time was not considered as an important parameter. Moreover, the low induced photocurrent $(110 \mu \mathrm{A})$ in this detuning system would not be sufficient to detune a larger coil. Furthermore, Fandrey et al have used a passive technique to detune of the coil and an optical modulator to transmit the RF detected signal to avoid any additional galvanic connection (Fandrey et al 2012). In contrast, Memis et al have worked with an active optical detuning circuit where a phototransistor associated with a battery instead of a PIN diode (Memis et al 2008). This proof of concept was however not suited to MRI applications due to its expected non-magnetic compatibility. Finally, as already mentioned, dStream ${ }^{\mathrm{TM}}$ architecture of Philips uses a galvanic active detuning method.

The overall objective of our project is to develop an endoluminal receiver coil devoid of any galvanic connection and excluded from any active component. The fully optical receiver coil has then to ensure two functionalities: the active optical detuning of the coil and the optical transmission of the detected NMR. The optical transmission was validated on an optical bench (Aydé et al 2013, Aydé et al 2014). In the following, the first functionality of the coil is presented. The optical detuning method was designed to ensure a reliable detuning based on the bias signal provided through optical fiber. After conception, the detuning circuit was associated with a single loop endoluminal coil with conventional NMR signal reception.

The coils and detuning circuits were electrically characterized on a measuring bench

before to be compared in vitro on a 3.0 T MR system in terms of signal uniformity and SNR.

\section{Methods}

\subsection{Coils Prototypes}

A rectangular single loop coil was designed using a $35 \mu$ m-thick copper trace (width $w=5.1 \mathrm{~mm}$, and length $l=47 \mathrm{~mm}$ on a FR4 substrate (relative permittivity $\epsilon_{r}=4$, thickness $t=0.8 \mathrm{~mm}$ ). Previous in vivo works (Armenean et al 2004, Beuf et al 2004, Pilleul et al 2005) have demonstrated that such size and geometry of the loop enable the depiction of bowel abnormalities by imaging the colon wall layers with an adequate penetration depth and a high in-plane spatial resolution. Figure 1 shows the photograph 


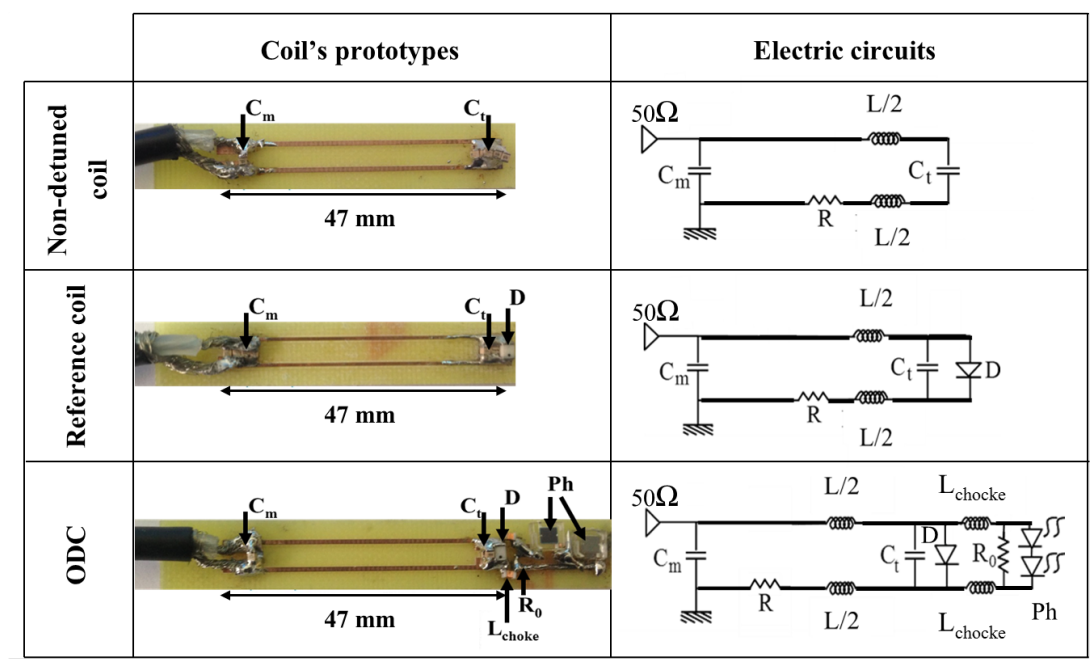

Figure 1. Photographs and electric circuits of the different endoluminal coil prototypes: non-detuned coil, reference coil, and ODC.

and the electric circuit of the different used coils. The endoluminal receiver coils were designed to operate with a discovery MR750 3.0 T MR System (GEHC, Milwaukee, USA). These coils are matched to $50 \Omega$ at a $127.73 \mathrm{MHz}$ frequency by using tuning and matching non-magnetic capacitors (American Technical Ceramics (ATC), New York, USA) on the distal and proximal parts of the coil.

A rectangular single loop without detuning circuit was first built. The adjustment of tuning and matching capacitors gives $C_{t}=34.4 \mathrm{pF}$ and $C_{m}=203.2 \mathrm{pF}$.

The galvanic-detuned coil (reference coil) can be carried out by adding a DH 80055 PIN diode (Temex Ceramics, Pessac, France) to the rectangular coil circuit in the distal part to ensure the detuning of the coil when activated. The tuning and matching capacitor values of this coil are respectively $C_{t}=36.3 \mathrm{pF}$ and $C_{m}=190 \mathrm{pF}$.

Similarly, the optical-detuned coil (ODC) was built by adding a PIN diode and two photodiodes to the rectangular coil. A silicon S6931-01 photodiode (Hamamatsu Photonics, Japan) was used, with a $6.6 \mathrm{~mm}^{2}$ photosensitive area. This photodiode is considered as "low-magnetic" based on MR images depicting very negligible local deformation in shape and intensity. Two photodiodes in series were used instead of one to ensure the requested voltage for a PIN diode to operate in direct mode. Tuning and matching capacitor values were $44.4 \mathrm{pF}$ and $206.2 \mathrm{pF}$ respectively. Two choke inductors of $1.2 \mu \mathrm{H}$ (SRT Resistor Technology, Germany) were added between the loop and the two photodiodes to block any alternating currents (AC) from interacting with the detuning circuit during NMR reception phase. When switching from detuning to tuning phase, the response of the coil is slow. Indeed parasitic capacitance of illuminated photodiodes enables photoelectrons to accumulate. Once light is off, these residual charges slowly flow out through the PIN diode which is still weakly biased (under threshold voltage) (Saleh et al 2007). Hence, a shunt resistance $R_{0}$ of $1.8 \mathrm{k} \Omega$ was added in parallel to force a rapid discharge of the photodiodes. The switching times form tuning to detuning and 


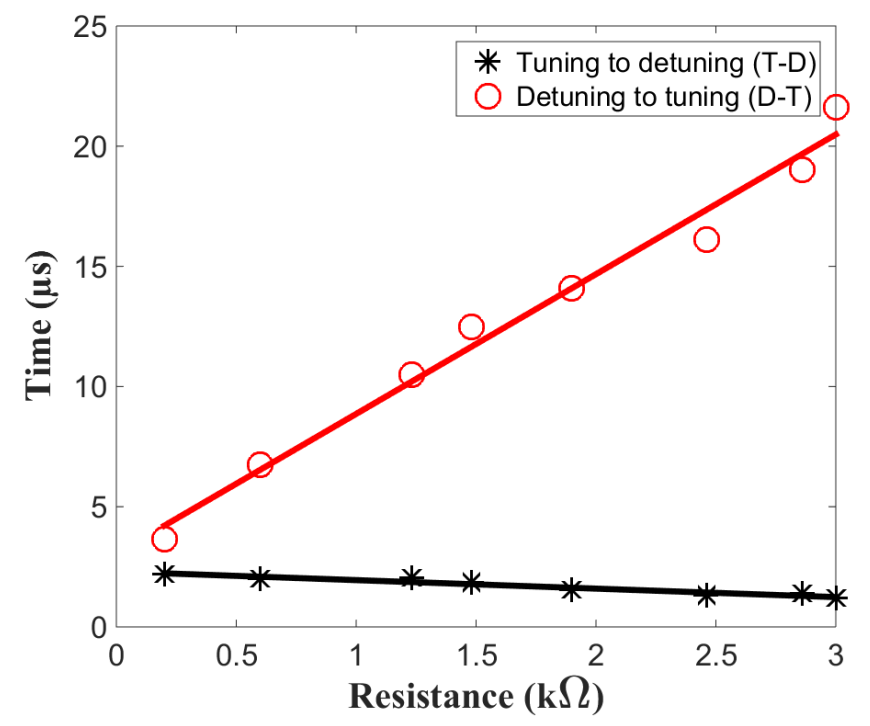

Figure 2. Switching time of the coil for tuning and detuning as a function of resistorvalue $R_{0}$.

reciprocally depend effectively on the electro-optical circuits and the added resistancevalue. For a high resistance value, the commutation time to tuning mode increases (Figure 2). On the other hand, the resistance should be sufficiently high to have the adequate voltage to activate the PIN diode. Taking these factors into consideration, an appropriate trade off was obtained for a resistance value of $1.8 \mathrm{k} \Omega$.

\subsection{Optical Detuning Unit}

In order to detune the coil, the two photodiodes added to the coil are illuminated by using two $30 \mathrm{~mW}$ fibred modulated laser diodes (Laser Components ${ }^{\circledR}$, Germany, $\lambda=$ $650 \mathrm{~nm}$ ). The optical detuning system begins when the MR system provides a bias DC current $(150 \mathrm{~mA})$ or a negative voltage to respectively detune the coil by turning on the laser diodes or tune the coil back by turning them off. With the connector used for single channel coil, the DC current is provided on the coaxial RF signal cable. A AC-DC filter was first placed to derive the DC bias signal. This DC bias signal was then used to drive the modulation of laser diodes. Given that these laser diodes must be powered and the electronic circuit could contain magnetic components, it was important to place the laser command circuit (including the laser diodes) distant enough from the MR system. To transmit the DC current, a coaxial cable cannot be used; because it is long enough to act as an antenna and depict signal from RF pulses. For this reason our solution was to convert directly the outgoing DC current from the filter to an optical signal using an electrical-to-optical conversion circuit, including an HFBR transmitter (Avago Technologies, 1404, $820 \mathrm{~nm}$, low optial power around $100 \mu \mathrm{W}$ ). The converted signal is transmitted via a multimode optical fiber (Radiospares, $\emptyset=0.2 \mathrm{~mm}$ ) to the laser command circuit. This latter consists of an optical to electrical converter: an HFBR 
a)

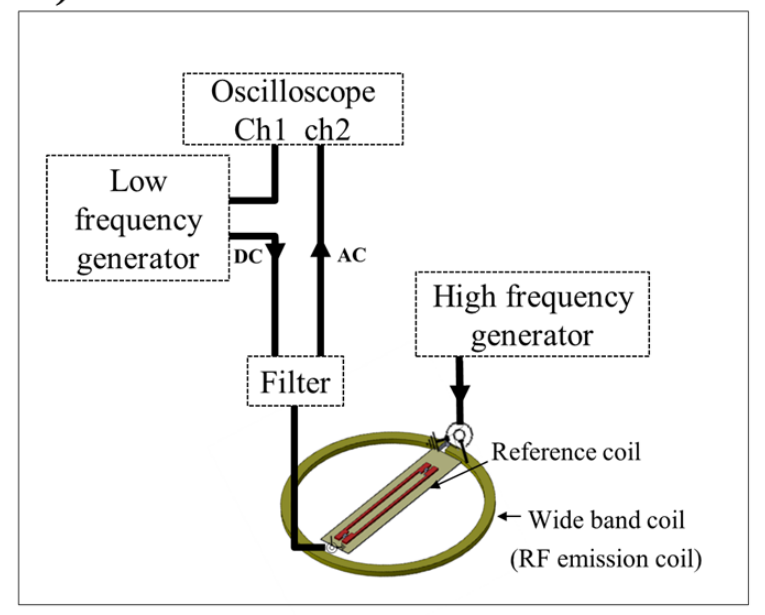

b)

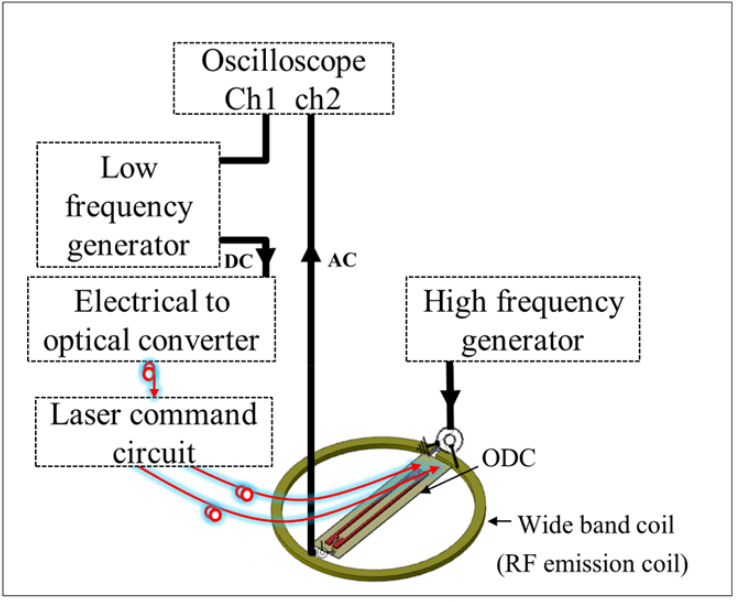

Figure 3. Schematic representation of switching time measurements procedure of an endoluminal coil added to a) a reference circuit and b) an ODC.

receiver (Avago Technologies, 2404, $820 \mathrm{~nm}$ ) followed by a non-inverting amplifier to modulate the states of the laser diodes and then decouple the coil.

\subsection{Experiments}

Coils were characterized using a vector network analyzer (VNA) (Agilent Technologies Inc., Santa Clara, CA, USA). The quality factor of each coil is determined from the reflection coefficient $\left|S_{11}\right|$ when the coils were loaded with a cylindrical phantom that will be later described in this section. $\left|S_{11}\right|$ is studied to assess detuning performance of each coil prototype. Furthermore, the switching time from tuned to detuned state of the coil and reciprocally was measured. The switching times were characterized on an experimental bench as presented in figure 3. A RF magnetic field was generated using a circular wide band RF emission coil excited by a high-frequency generator. The electrical power of the generator was $13 \mathrm{dBm}$ at a frequency of $127.73 \mathrm{MHz}$. The endoluminal receiver coil, resonating at the same frequency, was located in front of the RF emission coil to detect the magnetic field. The detuning of the reference coil was ensured by a low-frequency signal generator providing a square electrical $0 \mathrm{~V} / 5 \mathrm{~V}$ signal. This is equivalent to the decoupling signal from a MR System (Figure 3(a)). A filter was used here to visualize the DC and RF signals separately on the oscilloscope. On the other hand, the optical detuning was ensured by the optical detuning system presented in figure 3(b). The signal received by the endoluminal coils was measured using a numerical $500 \mathrm{MHz}$ oscilloscope (Agilent Technologies Inc., Santa Clara, CA, USA).

In vitro experiments were performed on a $3.0 \mathrm{~T}$ MRI. Figures 4(a) and 4(b) show the experimental setup. The coils were inserted into a plastic cylindrical phantom of 11 mm inner diameter, $90 \mathrm{~mm}$ outer diameter, and $100 \mathrm{~mm}$-long. The phantom was filled 
with a solution of $1.25 \mathrm{~g} \mathrm{NiSO} 4 \times 6 \mathrm{H}_{2} \mathrm{O}+5 \mathrm{~g} \mathrm{NaCl}$ per liter of distilled water to mimic the loading conditions of an internal coil inserted in a patient. First, the body coil was used as a transceiver to validate the efficiency of the detuning circuit by comparing the images acquired while using the tuned and the detuned coils. Corresponding images of the phantom without any endoluminal coil were also acquired to provide a basic reference. Two sequences were performed: a fast spin echo (FSE) sequence and a gradient echo sequence (GRE). Imaging parameters for the FSE sequence are $T R / T E=$ $3275 \mathrm{~ms} / 13.3 \mathrm{~ms}$, axial images with a $F O V=160 \times 160 \mathrm{~mm}^{2}$, slice thickness $=2 \mathrm{~mm}$, spacing between slices $=0.5 \mathrm{~mm}$, matrix $=256 \times 256$, receiver bandwidth $=16.67$ $\mathrm{kHz}$ or $\pm 8.335 \mathrm{kHz}$ (pixel receiver bandwidth $=65.11 \mathrm{~Hz} / \mathrm{Px}$ ). Image parameters for the GRE sequence are: $T R / T E=400 \mathrm{~ms} / 8.9 \mathrm{~ms}$, flip angle $=80^{\circ}$, axial images with a $F O V=160 \times 160 \mathrm{~mm}^{2}$, slice thickness $=2 \mathrm{~mm}$, spacing between slices $=0.5 \mathrm{~mm}$, matrix $=256 \times 256$, receiver bandwidth $=11.90 \mathrm{kHz}$ or $\pm 5.95 \mathrm{kHz}$ (pixel receiver bandwidth $=46.48 \mathrm{~Hz} / \mathrm{Px})$. The $\mathrm{SNR}$ calculation and the registration of the MR images were performed using MATLAB language (Mathworks, Milwaukee, USA). The SNR was determined by the mean of the signal intensity in the entire MR image divided by the mean standard deviation of a region of interest chosen outside the image of the phantom (in the air) which is the noise. For the registration, a self-made MATLAB program was developed to align MR images to a common coordinate system. For the purpose of quantifying the performance of the endoluminal coils in terms of SNR uniformity distribution, the same GRE and FSE sequences were applied, this time, using the endoluminal coil as a receiver. The comparison was done by drawing and identifying some specific SNR iso-contour lines in the images acquired by the different coils. Finally, the local variation of the temperature was measured for both reference coil and ODC used as receivers in the MR experiment. This measurement was performed using the optical fiber temperature system (Opsens, Canada). Fibers tips were mounted to the coil using Teflon rubber, and the whole was immersed in $1.5 \%$ agar-agar gel. For the reference coil, the fibers were placed at the proximal side of the loop, the distal (near the PIN diode), and the middle of the loop. For the ODC, the temperature variation was studied near the PIN diode and the two photodiodes.

\section{Results}

\subsection{Experimental Bench Measurement}

The reflection coefficient modulus $\left|S_{11}\right|$ as a function of the frequency is measured for each coil prototype in the tuned and detuned modes. The resonance frequency of the three coils is around $127.73 \mathrm{MHz}$. While the coil detuning, the resonance frequency is shifted except for the non-detuned coil. The quality factor $Q$ of the tuned coils is calculated on tuning mode following the procedure described in reference (Beuf et al 2006). The coil without detuning circuit has the highest quality factor $(Q=74)$. The quality factor decreases slightly for both reference coils and ODCs due to the added 
a)

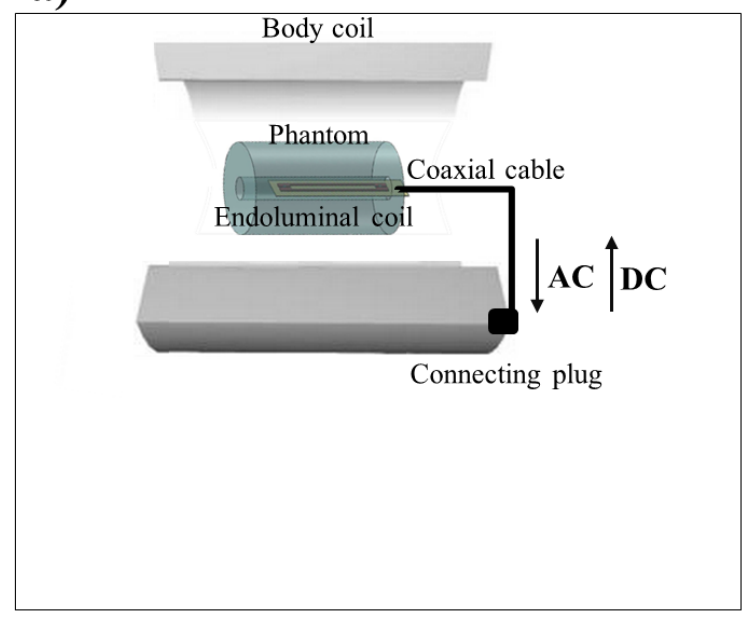

b)

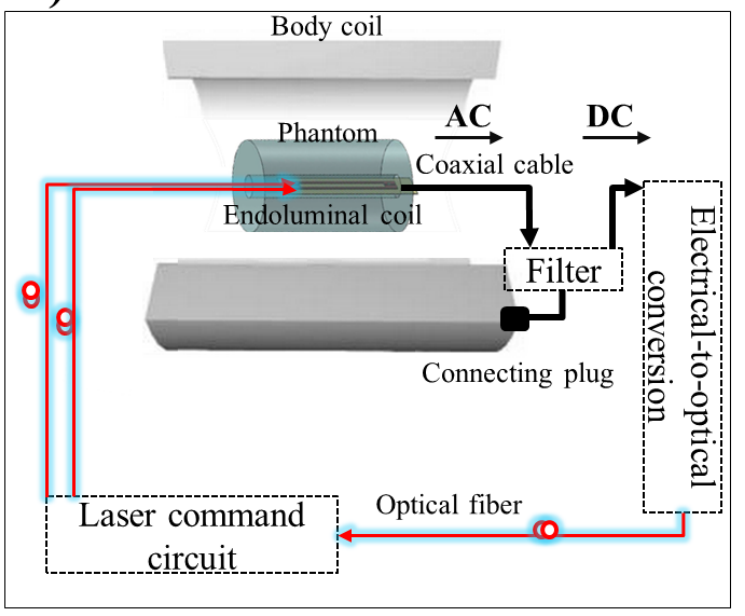

Figure 4. Schematic setups of coil detuning experiments performed on a $3.0 \mathrm{~T}$ MR system using an endoluminal coil with a) a galvanic-detuned circuit and b) an opticaldetuned circuit.

PIN diode and photodiode components. For the two coil prototypes considered in this work, the quality factors $Q$ are 53 and 55 respectively for the reference coil and ODC. On tuned mode, at $127.73 \mathrm{MHz},\left|S_{11}\right|=-39,33 \mathrm{~dB}$ and $\left|S_{11}\right|=-42,96 \mathrm{~dB}$ for coils with optical and galvanic detuning circuits respectively. While on detuned mode, at $127.73 \mathrm{MHz}$, the reflection coefficient of the coil with optical detuning system is close to $0 \mathrm{~dB}(-0.70 \mathrm{~dB})$; which is very similar to that of the reference coil $(-0.66 \mathrm{~dB})$. This result demonstrates that the current provided by the photodiodes is sufficient to detune the coil. Second, the switching times using the reference coil are fast compared to a typical RF pulse durations (in the order of milliseconds). Precisely, switching to tuning mode takes about $750 \mathrm{~ns}$, and switching back to detuning mode takes about $250 \mathrm{~ns}$. For the ODC, switching time is longer. By adding a resistance, the switching time to tuning state can decrease from approximatively $100 \mu$ s to $10 \mu$ s (Figure 5). Using the resistance-value mentioned above in the methods section $\left(R_{0}=1.8 \mathrm{k} \Omega\right)$, the switching times for ODC are $13.6 \mu \mathrm{s}$ and $1.7 \mu \mathrm{s}$ for tuning and detuning respectively.

\subsection{In vitro $M R$ Experiments}

The MR images acquired to evaluate detuning methods using the body coil as a transceiver are shown in figure 6 . For each acquisition, twenty-one axial images were acquired over the entire length $\mathrm{z}$ of the loop. The displayed axial signal intensity images and their SNR mapping corresponding to the central image out of stack. With the exception of the coil without detuning circuit, the signal magnitude of the MR images are very comparable with uniform signal distribution. Signal intensity and SNR maps corresponding to the non-detuned coil show clearly the non-uniform signal distribution close to the coil caused by the transmit $\mathrm{RF} \vec{B}_{1}$ field. The coil detuning is absolutely 
a)

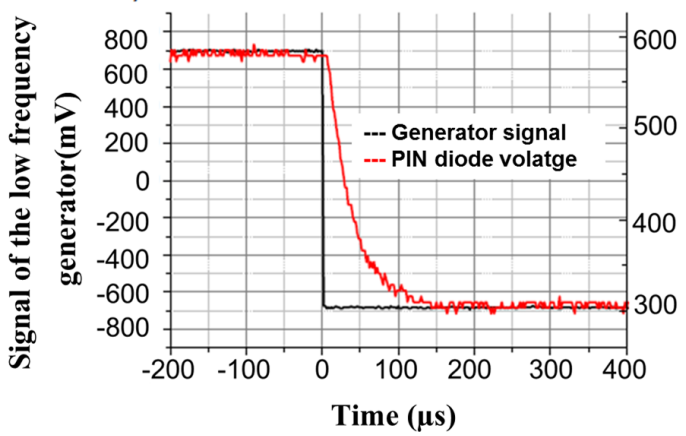

b)

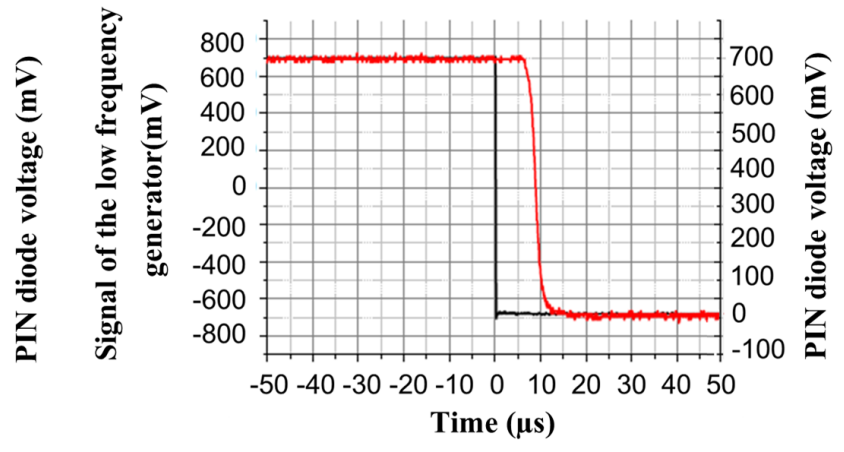

Figure 5. Response time graph showing the evolution of the voltage across the PIN diode versus time for ODC a) without and b) with resistor $R_{0}$.

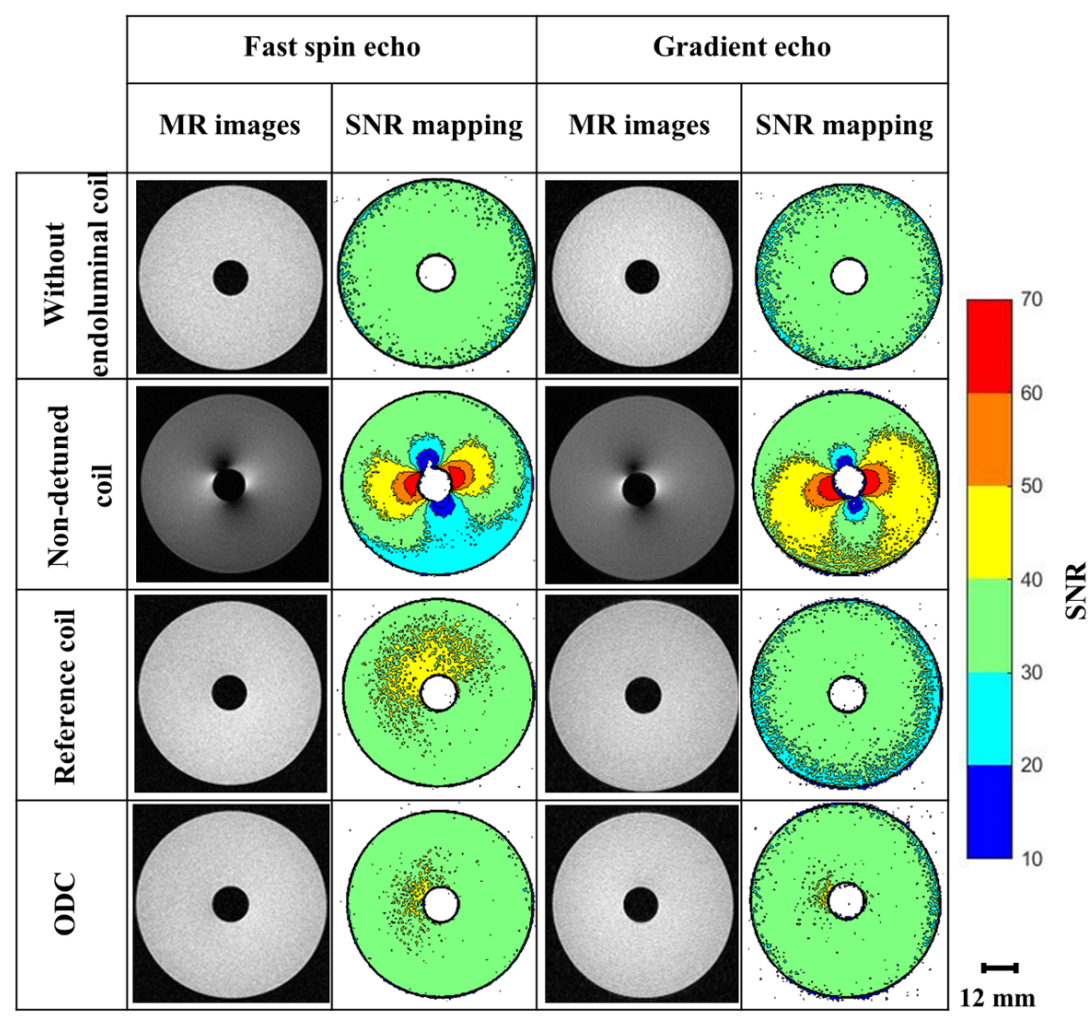

Figure 6. Axial images and SNR mapping of the phantom acquired using FSE and GRE sequence with the body coil used as a transceiver. The three endoluminal coils were located inside the phantom and they were continuously detuned.

mandatory.

The SNR values are ranging between 30 and 40 for the FSE and GRE sequences. For both sequences used, the SNR of MR images taken by the ODC with low-magnetic photodiodes are uniform and very comparable to the image of the phantom without any inserted coil. To study the SNR uniformity along the coil length $\mathrm{z}$, the mean SNR was measured in two square form regions of interest (ROI) of about $100 \mathrm{~mm}^{2}$ as shown in 


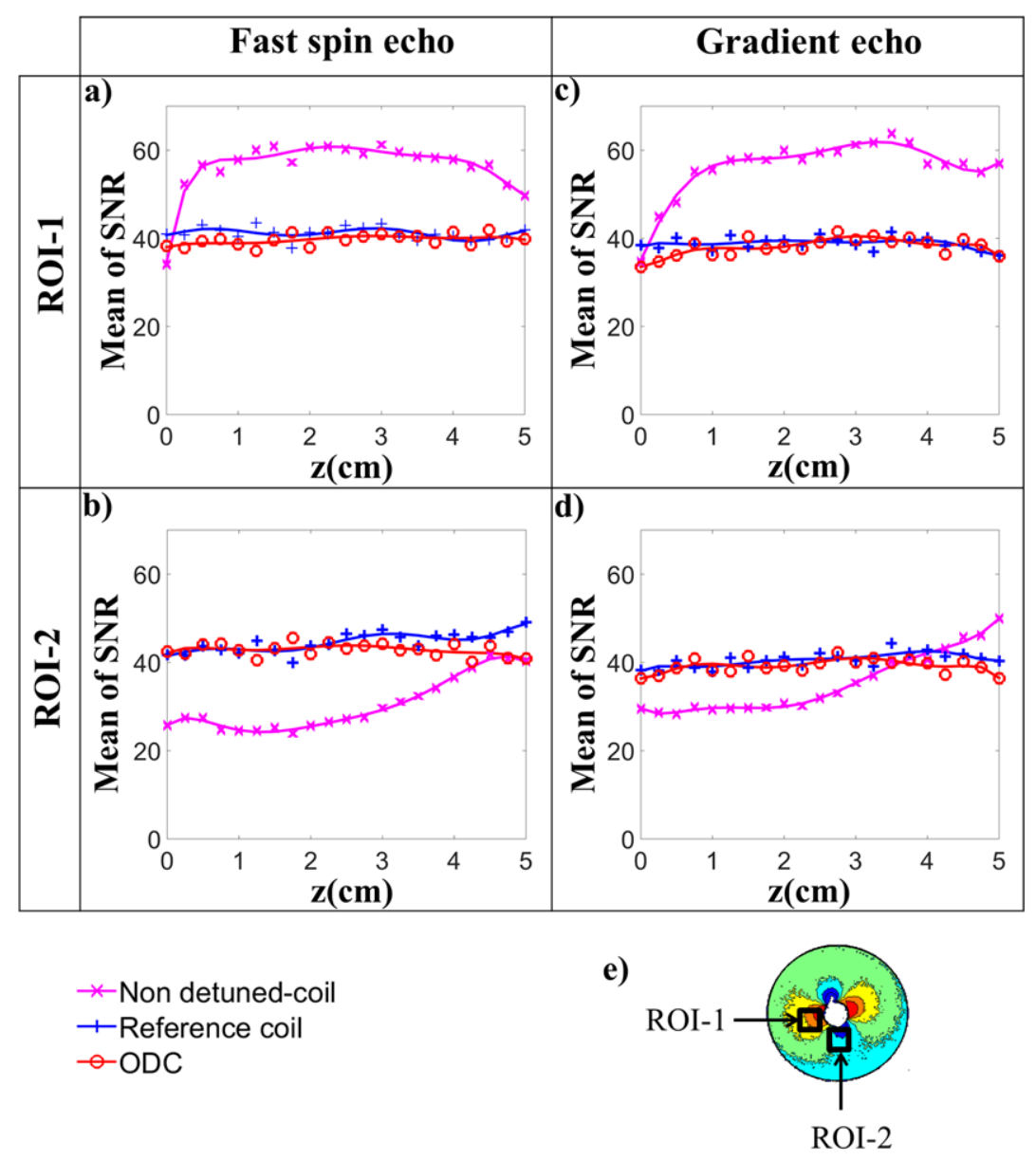

Figure 7. The measured signal-to-noise ratio of two regions of interest (ROI) for twenty-one axial images acquired by the body coil while using three different coils with (a-b) a fast spin echo sequence and (c-d) a gradient echo sequence. e) The choice of the positions of the two regions of interest (ROI-1 and ROI-2) is depending on the hyper and hypo signals presented in the MR image while using a non-detuned coil. The lines present a curve fitting to confirm the minimal variation of the SNR along the reference coil and the ODC.

figure 7. The positions of these two ROIs are identical for each image slice and they were chosen relatively to the positions of hyper and hypo signals of the image acquired while using a non-detuned coil (Figure 7(e)). The SNR uniformity was characterized by comparing the mean SNR of the two ROIs within each image of the stack. For the non-detuned coil, the figures 7(a) and 7(b) and figures 7(c) and 7(d) show that the SNR difference between the two ROI exceeds 30. For all detuned coils, SNR profiles along z for both ROIs are similar for the FSE and GRE sequences. It should be noted that for the ODC, the choice of the photodiode is important. It should have a small-size, a fast response time, and a low-magnetism. Figure 8 exhibits clearly that using magnetic photodiodes induce MR images distortions especially near the location of photodiodes.

The next experiment was done using the body coil as the RF transmitter and the endoluminal coil as a receiver. Given that the intensity of the image taken by a non- 
a)

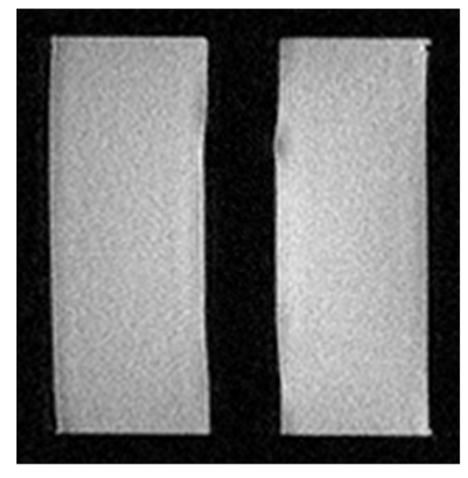

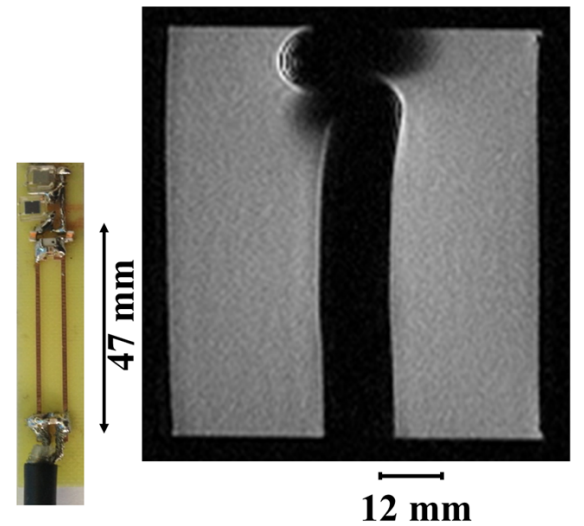

b)

Figure 8. Coronal images of the phantom using a) low-magnetic photodiodes, and b) magnetic photodiodes.

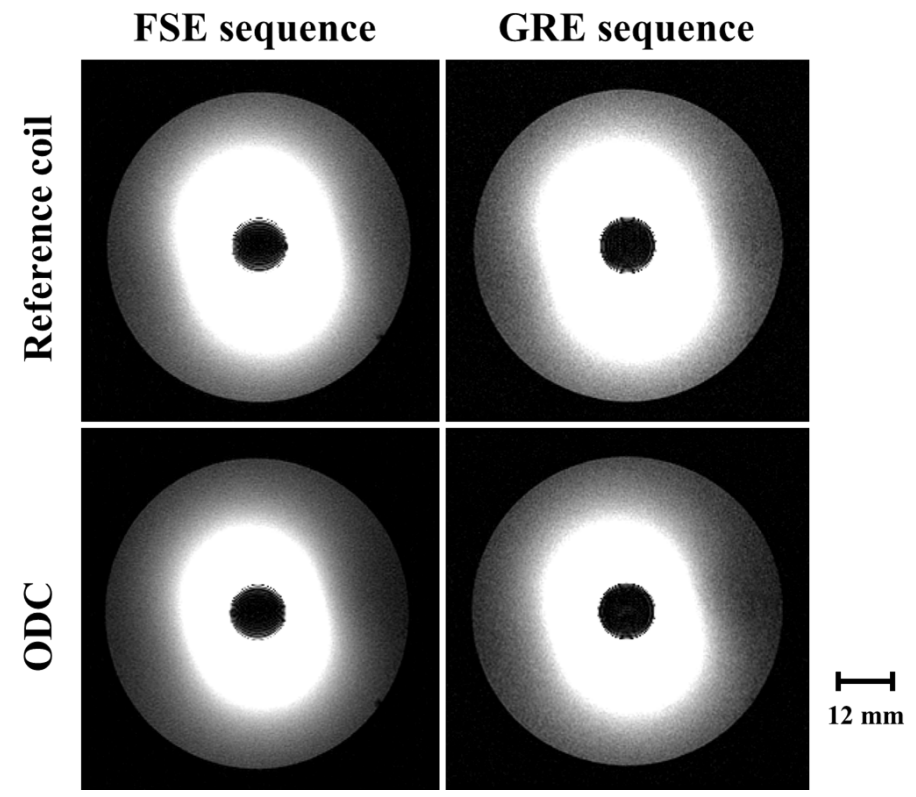

Figure 9. Axial MR images of the phantom acquired by the endoluminal coils.

detuned endoluminal coil is strongly altered by the cross-coil coupling, images were not acquired within these conditions. Images in figure 9 show comparable behavior between coils in terms of intensity and signal uniformity. According to figure 10, SNR iso-contours of the images taken with different coil prototypes are overlapping and this for both FSE and GRE sequences.

Finally, the variation of temperature for both the reference coil and ODC was measured during the in vitro experiments. For a galvanic detuning of the coil, the elevation of the temperature is around $2.77{ }^{\circ} \mathrm{C}$ near the PIN diode owing to the high detuning current emitted $(150 \mathrm{~mA})$. In the case of optical detuning, no change in temperature was discerned at the place of the PIN diode, this is due to the low 

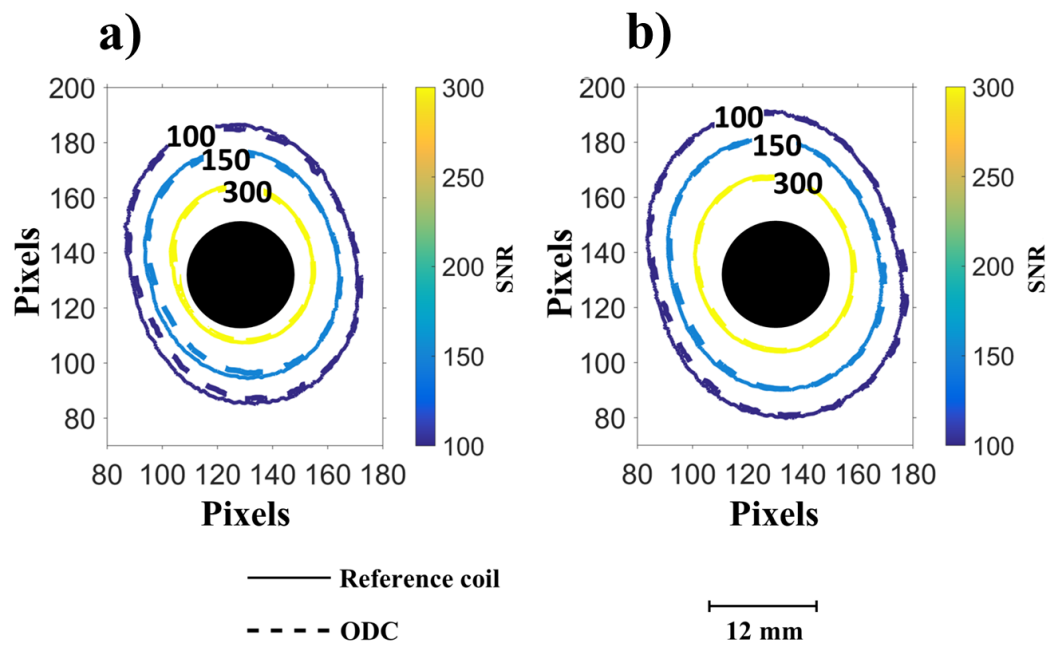

Figure 10. SNR iso-contours of the axial images acquired by the different coils using a) a FSE sequence and b) a GRE sequence.

photocurrent emitted $(11 \mathrm{~mA})$ by the photodiodes. And the negligible variation in the temperature $\left(\Delta T=0.24^{\circ} \mathrm{C}\right)$ near the illuminated photodiodes proves that the laser diodes $(30 \mathrm{~mW}, 650 \mu \mathrm{m})$ could be used without any potential risks to the imaged person. This result emphasizes that the use of optical detuning without RF induced heating effects could be considered as a safe active detuning mean.

\section{Discussion and conclusions}

The uniformity of signal intensity of images presented in figure 6 confirms that endoluminal ODC is as efficient as the galvanic-detuned coil (reference coil). The weak point of the ODC can be caused by the relatively low current injected into the PIN diode by the photodiodes. This current is actually around $11 \mathrm{~mA}$ which is relatively low compared to the $150 \mathrm{~mA}$ injected by the MR system to detune the reference coil. With this low intensity, no elevation of the temperature was recorded using the detuning circuit. But in order to fully ensure patient safety, the developed active detuning system should be associated with an endoluminal coil with a novel optical method of NMR signal transmission (Aydé et al 2013, Aydé et al 2014). A number of optical systems for NMR signal transmission have already been built (Fandrey et al 2012, Koste et al 2005, Memis et al 2008, Possanzini et al 2011, Yuan et al 2007). The associated detuning methods were either active and linked to MR console by a coaxial cable, or passive without any connection. In both cases, patient safety is very difficult to ensure for narrow endoluminal coil deeply inserted in the body. Other active optical detuning circuits were proposed for different MR applications (Korn et al 2011, Weiss et al 2004, Wong et al 2000). In the presented work, a PIN diode having very high impedance on blocking mode was used in parallel to tuning capacitor. The PIN diode was activated by two photodiodes being isolated from RF signals by two choke inductors. Using this 
detune method, $Q$ factor is not modified compared to that chosen by Weiss et al (2004) where a relatively low impedance photodiode non-isolated from the resonant circuit was used instead of the PIN diode. In the latter case, it was however not a real issue due to the pursued objectives of safe catheter tracking. Then an analysis of the behavior of the circuit in the reference (Korn et al 2011) shows that the switching time to tuning mode was not evaluated and probably not optimal. This would increase the switching time and limit temporal response of the receiver. With the use of a resistance $R_{0}$, the switching times of our circuit (around ten of $\mu \mathrm{s}$ at the slowest) are still quite small relative to the regular pulse duration (in the range of $\mathrm{ms}$ ) for most MR imaging sequences including fast and even ultra-fast imaging sequence such as echo-planar imaging. However, our circuit could not be fast enough for very specific sequence such as ultra-short echo time sequences (e.g. UTE). Moreover, in this work, a higher current was provided by the photodiodes to the PIN diode compared to optical detuning system of reference (Korn et al 2011) (11 mA > $100 \mu \mathrm{A})$ which is leading to more efficient detuning. Finally, unlike these previous works, our system configuration is suitable for all size of coil or loop. In fact, it can be easily adjusted in order to respond to all detuning applications (coils dimensions, tracking or imaging) by increasing the power of the laser or the radiant sensitive area dimension of the photodiode. It should be notice that in vivo studies of optical detuning is not adequate at this stage for several reasons. First, evaluating different detuning performances by comparing subtle changes with variable biological environments is not relevant. Second, keeping in mind that it is a safety issue, it would be unethical to perform this experiment in vivo. In conclusion, a novel compact active optical detuning circuit associated to a deported unit was developed and demonstrated with MRI endoluminal coil. The optical detuning circuit was based on the previous work of Korn et al (2011). The use of optical detuning was proposed to ensure total the patient safety. This strategy could be more widely developed and adapted in the future with multi-channel array coils. Optical-based detuning circuit associated with signal optical transmission via optical fibers could be the near future (Yuan et al 2016).

\section{Acknowledgment}

This work was supported by the Rhône-Alpes region (ADR and CIBLE projects) and the DGA (French Military Programs Management and Procurement Agency RAPID and SNIFER projects). This work was performed within the framework of LABEX PRIMES (ANR-11-LABX-0063) of Lyon University, within the program "Investissements d'Avenir" (ANR-11-IDEX-0007) operated by the French National Research Agency (ANR).

\section{References}

Armenean M, Beuf O, Pilleul F and Saint-Jalmes H, 2004 Optimization of endoluminal loop radiofrequency coils for gastrointestinal wall MR imaging IEEE Sensors J. 4 57-64 
Aydé R, Gaborit G, Jarrige P, Duvillaret L, Sablong R, Perrier A L and Beuf O 2013 Potentialities of an electro-optic crystal fed by nuclear magnetic resonant coil for remote and low-invasive magnetic field characterization IEEE Sensors J. 13 1274-80

Aydé A, Gaborit G, Dahdah J, Duvillaret L, Courjal N, Guyot C, Sablong R, Perrier A L and Beuf O 2014 Unbiased Electro-Optic waveguide as a sensitive nuclear magnetic resonance sensor IEEE Photon. Technol. Lett. 26 1266-69

Beets-Tan R G H, Lambregts D M J, Maas M, Bipat S, Barbaro B, Caseiro-Alves F, Curvo-Semedo L, Fenlon H M, Gollub M J, Gourtsoyianni S, Halligan S, Hoeffel C, Kim S H, Laghi A, Maier A, Rafaelsen S R, Stoker J, Taylor S A, Torkzad M R and Blomqvist L 2013 Magnetic resonance imaging for the clinical management of rectal cancer patients: recommendations from the 2012 European Society of Gastrointestinal and Abdominal Radiology (ESGAR) consensus meeting Eur. Radiol. $232522-31$

Beuf O, Pilleul F, Armenean M, Hadour G and Saint-Jalmes H 2004 In vivo colon wall imaging using endoluminal coils: feasibility study on rabbits J. Magn. Reson. Imaging 20 90-96

Beuf O, Jaillon F and Saint-Jalmes H 2006 Small-animal MRI: signal-to-noise ratio comparison at 7 and $1.5 \mathrm{~T}$ with multiple-animal acquisitions strategies Magn. Reson. Mater. Phy. 19 202-8

Burl M and Zou M X 2005 Transmit mode coil detuning for MRI systems US6850067B1

Detti V, Grenier D, Perrin E and Beuf O 2011 Assessment of radiofrequency self-heating around a metallic wire with MR T1-based thermometry Magn. Reson. Med. 66 448-55

Dewhurst C E and Mortele K J 2013 Magnetic resonance imaging of rectal cancer Radiol. Clin. of N. Am. 51 121-31

Dorez H, Sablong R, Canaple L, Saint-Jalmes H, Gaillard S, Moussata D and Beuf O 2016 Endoluminal high-resolution MR imaging protocol for colon walls analysis in a mouse model of colitis Magn. Reson. Mater. Phy. 29 657-69

Fandrey S, Weiss S and Muller J 2012 A novel active MR probe using a miniaturized optical link for a 1.5-T MRI scanner Magn. Reson. Med. 67 148-55

Geenen R W F, Hussain S M, Siersema P D, Poley J W, Kuipers E J and Krestin G P 2007 Current status of MRI in patients with inflammatory bowel disease colitis Appl. Radiol. 36 10-22

Giusti S, Buccianti P, Castagna M, Fruzzetti E, Fattori S, Castelluccio E, Caramella D and Bartolozzi C 2012 Preoperative rectal cancer staging with phased-array MR Radiat. Oncol. 7 1-10

Kantor $\mathrm{H}$, Briggs $\mathrm{R}$ and Balaban $\mathrm{R} 1984$ In vivo ${ }^{31} \mathrm{P}$ nuclear magnetic resonance measurements in canine heart using a catheter-coil Circulation Research 55 261-6

Korn M, Umathum R, Schulz J, Semmler W and Bock M 2011 Optically detunable, inductively coupled coil for self-gating in small animal magnetic resonance imaging Magn. Reson. Med. 65 882-8

Koste G P, Nielsen M C, Tolliver T R, Frey R L and Watkins R D 2005 Proc. ISMRM, 13th Scientific Meeting and exhibition(Florida) (USA: ISMRM) p 411.

Ladd M E, and Quick H H 2000 Reduction of resonant RF heating in intravascular catheters using coaxial chokes Magn. Reson. Med. 43 615-9

Memis O G, Eryaman Y, Aytur O and Atalar E 2008 Miniaturized fiberoptic transmission system for MRI signals Magn. Reson. Med. 59 165-73

Peterson D M, Beck B L, Duensing G R, Fitzsmmons J R 2003 Common mode signal rejection methods for MRI: reduction of cable shield currents for high static magnetic field systems Concepts Magn. Reson. Part B (Magn. Reson. Eng.) 19B 1-8

Pilleul F, Beuf O, Godefroy C, Scoazec J Y, Armenean M, Armenean C, Perrin E, Valette P J and Saint-Jalmes H 2005 High-resolution MR imaging appearance of colonic tissue in rabbits using an endoluminal coil Magn. Reson. Mater. Phy. 18 238-44

Possanzini C, Harvey P R, Ham K, Hoogeveen R and Stoesz M 2011 dStream Architecture, The digital revolution in MRI http://www.healthcare.philips.com

Ramgolam A, Sablong R, Lafarge L, Saint-Jalmes H and Beuf O 2011 Optical spectroscopy combined with high-resolution magnetic resonance imaging for digestive wall assessment: endoluminal bimodal probe conception and characterization in vitro on organic sample and in vivo on a rabbit 
J. Biomed. Opt. 16 117005-13

Saleh B E A and Teich M C 2007 Fundamentals of Photonics (USA: 2nd ed. John Wiley \& sons Inc.)

Torkzad M R, Pahlman L and Glimelius B 2010 Magnetic Resonance Imaging (MRI) In rectal cancer: a comprehensive review Insights Imaging 1 245-267

Weiss S, Kuehne T, Brinkert F, Krombach G, Katoh M, Schaeffter T, Guenther R W and Buecker A 2004 In vivo safe catheter visualization and slice tracking using an optically detunable resonant marker Magn. Reson. Med. 52 860-8

Weiss S, Vernickel P, Schaeffter T, Schulz V and Gleich B 2005 Transmission line for improved RF safety of interventional devices Magn. Reson. Med. 54 182-9

Wong E Y, Zhang Q, Duerk J L, Lewin J S and Wendt M 2000 An optical system for wireless detuning of parallel resonant circuits J. Magn. Reson. Imaging 12 632-8

Wright A C, Song H K and Wehrli F W 2000 In vivo MR micro imaging with conventional radiofrequency coils cooled to 77K Magn. Reson. Med. 43 163-9

Yeung C J, Susil R C and Atalar E 2002 RF safety of wires in interventional MRI: using a safety index Magn. Reson. Med. $47187-93$

Yuan J, Wei J and Shen G X 2007 A direct modulated optical link for MRI RF receive coil interconnection J. Magn. Reson. 189 130-8 